\title{
Entropy-Driven Selectivity for Chain Scission: Where Macromolecules Cleave ${ }^{* *}$
}

\author{
Kai Pahnke, Josef Brandt, Ganna Gryn'ova, Ching Y. Lin, Ozcan Altintas, Friedrich G. Schmidt, Albena \\ Lederer, ${ }^{*}$ Michelle L. Coote, ${ }^{*}$ and Christopher Barner-Kowollik*
}

\begin{abstract}
We show that, all other conditions being equal, bond cleavage in the middle of molecules is much more favored compared to bond cleavage at the end. Multiple experimental and theoretical approaches have been used to study the selectivity for bond cleavage or dissociation in the middle vs. the end of both covalent and supramolecular adducts and the extensive implications for other fields of chemistry including, e.g., chain transfer, polymer degradation or control agent addition are discussed. The observed effects which are a consequence of the underlying entropic factors were predicted on the basis of simple theoretical models and demonstrated via high temperature (HT) NMR spectroscopy of self-assembled supramolecular diblock systems as well as temperature dependent size exclusion chromatography (TD SEC) of covalently bonded DielsAlder step-growth polymers.
\end{abstract}

[*] K. Pahnke, Dr. O. Altintas, Prof. C. Barner-Kowollik Preparative Macromolecular Chemistry Institut für Technische Chemie und Polymerchemie Karlsruhe Institute of Technology (KIT)

Engesserstr. 18, 76131 Karlsruhe (Germany)

E-mail: christopher.barner-kowollik@kit.edu

K. Pahnke, Dr. O. Altintas, Prof. C. Barner-Kowollik Institut für Biologische Grenzflächen

Karlsruhe Institute of Technology (KIT)

Hermann-von-Helmholtz-Platz 1, 76344 Eggenstein-Leopoldshafen (Germany)

J. Brandt, Dr. Albena Lederer

Leibniz-Institut für Polymerforschung Dresden

Hohe Strasse 6, 01069 Dresden (Germany)

E-mail: lederer@ipfdd.de

J. Brandt, Dr. Albena Lederer

Technische Universität Dresden

01062 Dresden (Germany)

Dr. G. Gryn'ova, Dr. C. Y. Lin, Prof. M. L. Coote

ARC Centre of Excellence for Electromaterials Science

Research School of Chemistry

Australian National University (ANU)

Canberra, ACT 0200 (Australia)

E-mail: michelle.coote@anu.edu.au

Present address of G. G.: Ecole polytechnique fédérale de Lausanne (Switzerland)

Dr. F. G. Schmidt

Evonik Industries AG

Paul-Baumann-Strasse 1, 45764 Marl (Germany)

${ }^{* *}$ C. B.-K., M. L. C. and A. L. are grateful for continued support from and the excellent collaboration with Evonik Industries. C. B.-K. additionally acknowledges funding from the KIT via the Helmholtz association (BIFTM and STN programs) as well as the German Research Council (DFG). M.L.C. acknowledges generous allocations of supercomputing time on the National Facility of the National Computational Infrastructure and an Australian Research Council Future Fellowship.

Supporting information for this article is given via a link at the end of the document.
Reversible covalent and supramolecular bonding systems are increasingly being employed for the generation of materials with novel characteristics such as self-healing or otherwise stimuliresponsive abilities, self-assembly into complex molecular architectures, mimicking of proteins, easily recyclable substructures or self-immolative polymers. ${ }^{[1]}$ In such dynamic systems, a thorough understanding of the numerous factors that influence the binding constants is very important. ${ }^{[2]}$ Moreover, it is not only the binding characteristics of reversible ligation sites that have to be closely considered, but also processes that are less dynamic, as they may be impaired by similar restrictions. Recently, we demonstrated the significance of physical molecular properties in addition to the known chemical effects on reversible and irreversible association or degradation. It was predicted theoretically and demonstrated in diverse experiments that, due to the differences in released entropy, a mass and chain length effect leads to favored debonding, dissociation and degradation of heavier or longer species, and, furthermore, the intramolecular mobility (i.e., chain stiffness) also influences reaction equilibria. ${ }^{[3]}$ While this "entropy effect" has so far been utilized to alter reaction properties in self-healing materials, by its nature it is in principle applicable to all chemically-controlled reactions of macromolecular species and must be considered when small molecular chemistry is transferred to larger systems. In the light of these demonstrated entropic effects on bonding or association equilibria, an obvious question is whether, all other factors being equal, the position of a bonding or debonding site in a given molecule could affect the reaction equilibrium. To address this question, in the present work a set of experiments and calculations with a special focus on the selectivity for bond cleavage in the middle vs. at the chain end was considered (Scheme 1)

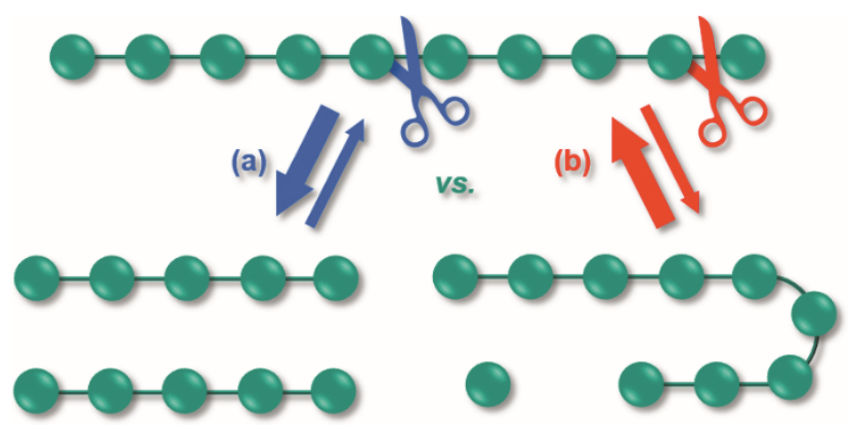

Scheme 1. Schematic view of the theoretical and experimental setup to determine characteristic differences between bond cleavage (a) in the middle and (b) at the end of a molecule.

As a first proof of concept, we considered a highly simplified chemical model of a polymer, comprising a linear string of point 
masses with arbitrary, yet realistic dimensions. Using standard textbook equations, we calculated the translational $\left(\Delta S_{\text {trans }}\right)$ and rotational $\left(\Delta S_{\text {rot }}\right)$ contributions to the entropy of bond cleavage at either the middle or the end of the chain under otherwise identical conditions (Table 1).

Table 1. Values for the translational and rotational entropy ${ }^{[a]}$ for de-bonding of simple geometric models determined via statistical thermodynamics methods.

\begin{tabular}{ccccc}
\hline $\begin{array}{c}\text { Cleavage } \\
\text { in/at the } \ldots\end{array}$ & \multicolumn{2}{c}{$\ldots$ middle: } & \multicolumn{2}{c}{$\ldots$ end: } \\
\hline initial & 148.9 & 82.4 & 136.1 & -2.6 \\
longer & 148.9 & 87.6 & 136.1 & -0.7 \\
heavier & 157.5 & 99.3 & 137.4 & -0.3 \\
\hline
\end{tabular}

[a] $\Delta S$ in $\mathrm{J} \mathrm{mol}^{-1} \mathrm{~K}^{-1}$ at $298 \mathrm{~K}$.

Full details of the model used and the calculations are provided in the Supporting Information (SI). Our choice of a simplified model allowed us to consider much larger chains than would otherwise be possible, and to study the broader chemical trends on these parameters in the absence of the complicating effects of the local chemical nature of the employed ligation sites. Values of the vibrational entropy $\left(\Delta S_{\text {vib }}\right)$ and enthalpy $(\Delta H)$ were not calculated because, in contrast to $\Delta S_{\text {trans }}$ and $\Delta S_{\text {rot, }}$, they are both highly sensitive to the local chemical nature of the employed ligation sites and relatively insensitive to chain length effects. ${ }^{[3 e]}$ From Table 1 it is clear that, in all cases, cleavage in the middle of the chain is strongly favored entropically (i.e., greater positive $\Delta S$, compare $\Delta G=\Delta H-T \Delta S$ ) due to the reinforcing effects of translational and rotational entropy. Moreover, this preference becomes more pronounced as the chains become heavier and/or longer. In other words, the chain length and mass effects on $\Delta S$ are much more pronounced for cleavage in the middle of a polymer chain, than for the equivalent reaction at the chain end, with the former expected to converge much less slowly as a function of chain length than the latter. One immediate consequence of these results is the prediction that rate and equilibrium constants for polymerization reactions in which a growing polymeric species adds to a small molecule (as in chain polymerization) should converge much more quickly with chain length than those in which two growing polymeric species adds to each other. Indeed, it is well known that the propagation rate coefficient in free-radical polymerization converges rapidly with chain length $(<1000 \mathrm{u}),{ }^{[4]}$ whereas as noted above our experimental studies have demonstrated that chain length and mass effects on the equilibrium constant for step-growth DielsAlder (DA) polymers extends to high molecular weights (ca. $10^{5} \mathrm{u}$ ). ${ }^{[\mathrm{b}]}$ As the use of simple geometric models can only account for the qualitative trends, the experimental investigation of actual chemical systems is needed to provide a deeper insight into the quantitative characteristics of the position effect. Therefore, we next considered the examination of the temperature dependent association of different supramolecular diblocks via high temperature (HT) NMR spectroscopy (see SI for synthetic details). While entropic effects are a generally applicable principle and thus not exclusive for polymer systems, such systems allow for a facile adjustment of the molecular parameters in question. A pair of diblocks, each containing two blocks of differing individual, yet approximately same overall length, was self-assembled in an aprotic solvent (tetrachloroethane- $\mathrm{d}_{2}, c=6 \mathrm{mmol} \mathrm{L}^{-1}$ ) via cyanuric acid (CA) and Hamilton wedge (HW) recognition units to resemble dynamic non-covalent association in the middle or at the end of a macromolecule (Figure 1 (a) and (b)), enabling the examination of the two extreme examples when investigating the effect of the position of a ligation site within a molecule.

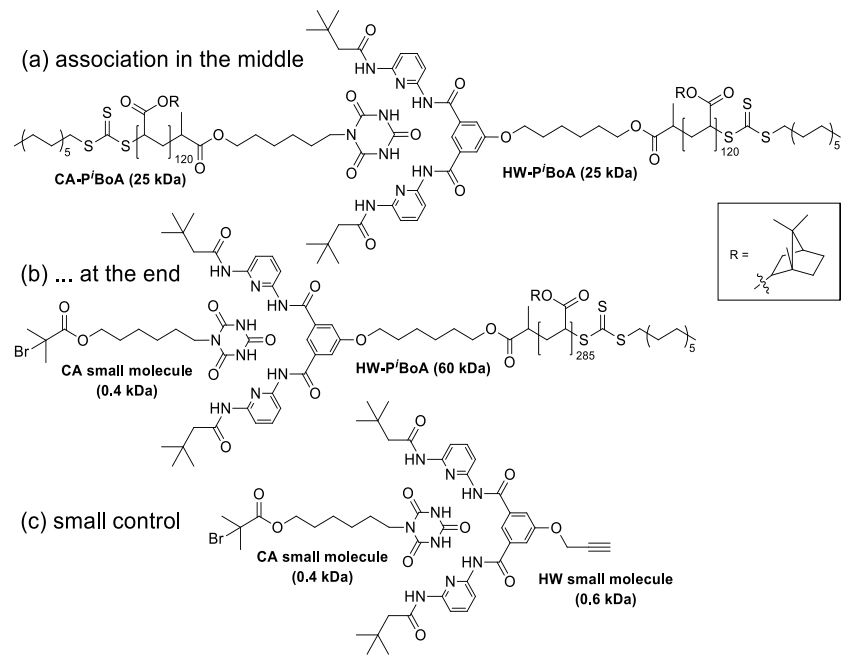

Figure 1. The supramolecular building block pairings with (a) poly(isobornyl acrylate) moieties of $M_{\mathrm{n}, \mathrm{SEC} / \mathrm{NMR}}=25 \mathrm{kDa}$ on both sides of the ligation site, (b) one small molecular CA unit in combination with a HW functional poly(isobornyl acrylate) of $M_{\mathrm{NMR}}=60 \mathrm{kDa}$ and (c) the small molecular control test.

The bonding in the middle system in combination with a readily available small molecular control comprising identical recognition units (Figure 1 (c)) were already studied under said conditions in a previous study and are indicated for comparison. ${ }^{[3 \mathrm{c}]}$ Subsequently, the chemical shift values of the CA imide protons enabled the qualitative observation of the temperature dependent degree of association due to their equilibrium dependent resonance with higher downfield shift values being related to a higher degree of association (Figure 2 and SI, HT NMR Spectra). ${ }^{[1], 3 c, 5]}$ Competing interactions of acrylate backbones with the applied hydrogen bonding motifs were excluded via both intramolecular spacers and measurements of small molecular recognition units in the presence of non-functional polyacrylates. ${ }^{[3 c, 6]}$ For a quantitative assessment of equilibrium constants, NMR titration experiments were conducted to determine the dissociation constants of the associated individual block pairings (see SI, NMR Titration). ${ }^{[7]}$ To circumvent problems of signal isolation due to unfavorable exchange rates or overlapping resonances, a temperature of $100^{\circ} \mathrm{C}$ was applied. In accordance with the theoretical considerations, it could be shown that, at a given temperature, dissociation in the middle 
$\left(K_{\text {diss,middle }}=(3.7 \pm 0.15) \cdot 10^{-3}\right)$ is favored by a factor of 2 in comparison with dissociation of a small molecule at the end of a macromolecule $\left(K_{\text {diss,end }}=(1.9 \pm 0.15) \cdot 10^{-3}\right)$. Thus, dissociation from the end is comparably unfavorable as the dissociation of two small molecules $\left(K_{\text {diss,small }}=(1.8 \pm 0.15) \cdot 10^{-3}\right)$ due to the altered association constants. ${ }^{[3 \mathrm{c}]}$

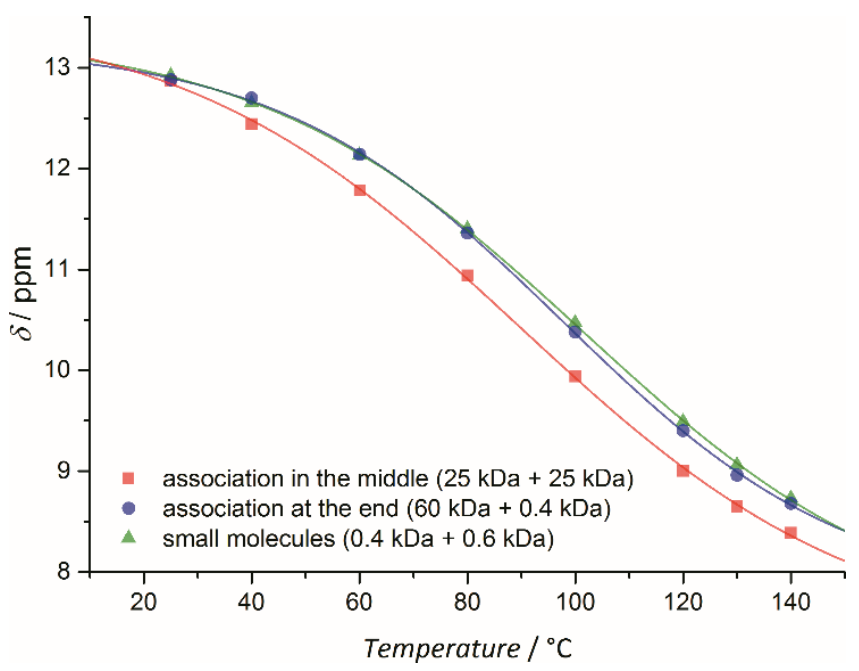

Figure 2. Temperature dependent $C A$ imide proton resonance in $\mathrm{C}_{2} \mathrm{D}_{2} \mathrm{Cl}_{4}$ for building blocks associated via $\mathrm{CA}$ and $\mathrm{HW}$ recognition units in the middle (ם) or at the end $(\mathcal{)})$ of macromolecules as well as for small molecular adducts $(\star)$, indicating higher degrees of association for higher downfield shift values.

Remarkably, even though large polymer systems were employed, the position effect has not leveled-off yet by virtue of a possibly overlapping mass effect, confirming the theoretical prediction of a much higher impact of molecular parameters on central bonding sites and emphasizing the significance of the findings. To gain further knowledge regarding the versatility and specificity of the entropic position effect, an alternative experimental approach was realized via hetero DA step-growth polymers of a cyanodithioester (CDTE) difunctional small molecular linker and cyclopentadiene (Cp) difunctional macromolecular building blocks comprising poly(methyl acrylate) (PMA), poly(isobornyl acrylate) ( $\left.P^{i} B o A\right)$ as well as polystyrene (PS) backbones (Figure 3, see SI for synthetic details).

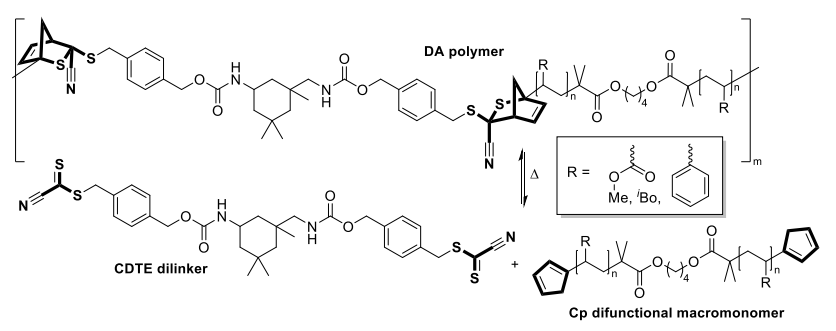

Figure 3. Reaction scheme of the thermoreversible degradation of DA stepgrowth polymers out of cyclopentadiene difunctional macromolecular building blocks and a cyanodithioester difunctional linker.
We experimentally investigated their retro $\mathrm{DA}(\mathrm{rDA})$ degradation via diverse temperature dependent size exclusion chromatography (TD SEC) experiments. ${ }^{[8]}$ Exemplary results are shown for DA-PMA systems, for additional results (PS and $\mathrm{P}^{\mathrm{B} B \mathrm{~A}}$ systems) refer to the SI. In TD SEC, the DA-PMA was measured in situ at $90^{\circ} \mathrm{C}$, resulting in a chromatogram corresponding to the molecular weight distribution as depicted in Figure 4 (a), evidencing the beginning of a cleavage process due to the starting rDA reaction (compare Figure S1 for an initial SEC trace at ambient temperature).

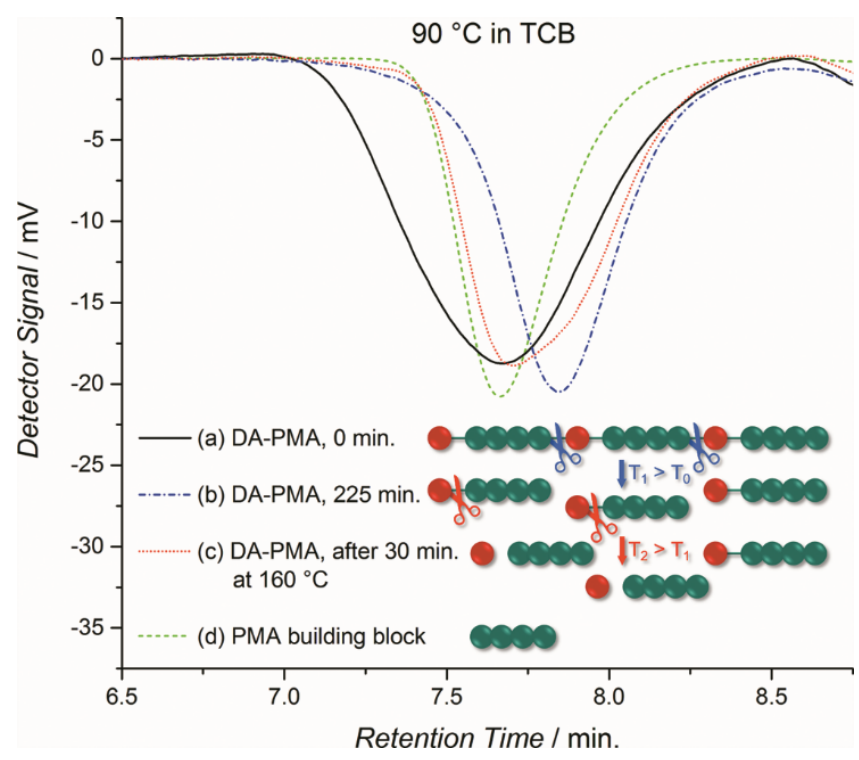

Figure 4. TD SEC chromatogram of a thermoreversible DA-PMA at $90{ }^{\circ} \mathrm{C}$ and $160{ }^{\circ} \mathrm{C}$ and its corresponding building block in 1,3,5-trichlorobenzene (TCB) as well as a schematic view of the degradation process of such DA step-growth polymers, consisting of macromonomers (green) and a small molecular dilinker (red), at increasing temperatures, here $\mathrm{T}_{0}=$ ambient temperature, $\mathrm{T}_{1}=90^{\circ} \mathrm{C}$, $\mathrm{T}_{2}=160^{\circ} \mathrm{C}$

After $225 \mathrm{~min}$. at $90^{\circ} \mathrm{C}$, a second chromatogram of the same sample was acquired (Figure 4 (b)) which shows the expected shift to higher elution volumes, indicating decreasing molecular weight due to the rDA reaction. It should be expected that the lowest achievable weight represents the chromatogram of the isolated polymer building block (Figure $4(d)$ ). However, the released species elutes at even higher elution times, representing irrationally low molecular weights. While thermal decomposition could not be evidenced, in liquid chromatography high elution volumes are observed if enthalpic interactions with the column material, originating from highly polar functional groups, lead to additional retention. ${ }^{[9]}$ In fact, such behavior could be attributed to the employed CDTE linker and is also the reason for the apparent occurrence of low molecular weight material in the DA polymer (Figure 4 (a)): A polymer building block that is still bound to one (or two) very polar linker units is thus expected to elute later than a block without linker molecules, although both can have very similar molar masses. To proof this hypothesis and deliberately utilize it for an assessment of the question where molecules cleave first, the very same DA polymer sample was heated to $160^{\circ} \mathrm{C}$ in order to drive the rDA reaction further, and was then 
measured at $90{ }^{\circ} \mathrm{C}$ to achieve similar measurement conditions. The obtained chromatogram (Figure 4 (c)) clearly shows a shift of the peak, converging into the shape of the building block signal (Figure $4(\mathrm{~d})$ ), thus proving that only at higher rDA conversions the bonds at the end are cleaved. This behavior can be ascribed to the favored scission of bonds in the middle of molecules, while the release of small species at terminal binding sites is disfavored. Similar results could be obtained for a DA-PS (refer to Figure S6 in the SI). Additional analysis of the decrease of DA-PMA in comparison with the release of the dilinker via deconvolution of chromatograms with suppressed interactions of the polar CDTE linker and the column material via adjusted solvent mixtures confirms these assumptions (see SI for experimental details). As the linker signals can now be isolated in the applied solvent mixture, not only the temperature dependent decrease of the concentration of the DA polymer species, but also the release of the CDTE linker can be calculated (Figure 5).

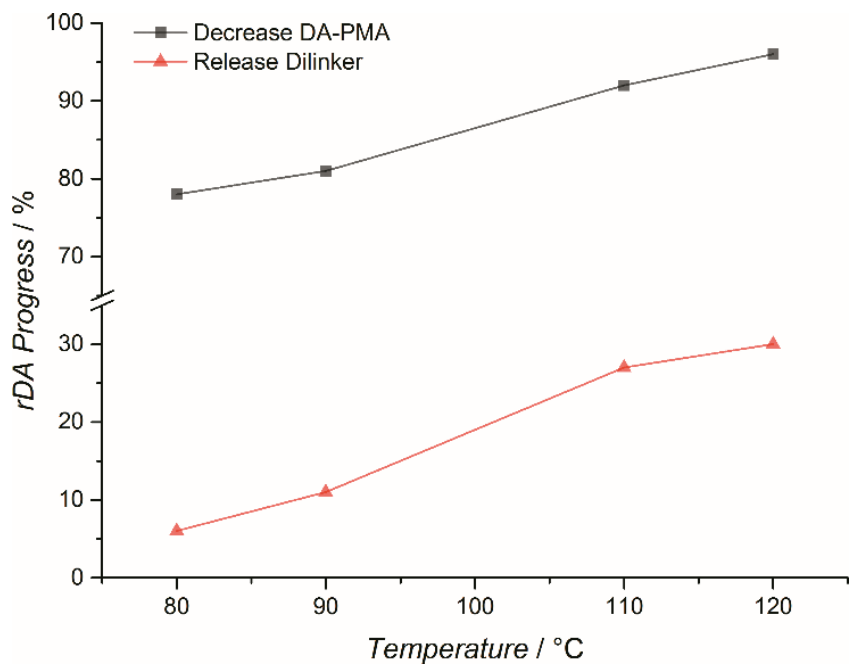

Figure 5. Temperature dependent decrease of DA-PMA in comparison with the release of the DA dilinker.

In contrast to a purely statistical degradation, it is clearly visible that - while the DA polymer debonds very fast - only at high temperatures significant amounts of the linker species can be detected. Analogous observations are made for the DA-PiBoA systems (refer to Figure S4 in the $\mathrm{SI}$ ) and via the inclusion of a UV detector for DA-PS systems (refer to Figure S6 in the SI), thus completely supporting the herein described effects.

In conclusion, we demonstrated that debonding in the middle of molecules is favored in comparison to debonding at the end for both covalent and non-covalent interactions (Scheme 1). Clearly, these findings can be exploited to tune reaction equilibria of known dynamic chemistries and need to be considered when new reversible ligation sites are transferred to other systems. Moreover, being properties of polymer chains rather than the specific chemical reaction involved, these entropic effects are not limited to dynamically bonding self-healing polymers but should have general applicability when considering chemical reactions involving macromolecular species. Among others, extended chain length effects on polymerization rate coefficients are not necessarily restricted to reactions occurring under diffusion control, but need to be considered in chemically-controlled bimolecular reactions involving two polymeric species. Such examples could thus include polymer degradation, chain transfer to polymer, or control agent addition. In the case of thermal or oxidative polymer degradation, no clear trends besides a mass effect could be deduced from literature, probably due to the generation of radicals via a manifold of reaction channels. ${ }^{[3 d, 10]}$ Thus, for example, an entropic tendency to undergo mid-chain homolysis is likely countered by the greater preponderance of unsaturated initiation sites at the chain end. ${ }^{[11]}$ Nonetheless, ultrasonication or general shearing of polymers leads to a favored chain scission in the middle of the molecule. ${ }^{[12]}$ In the case of controlled radical polymerization, this work suggests that, unlike low molecular weight control agents, polymeric control agents are likely to become less reactive to growing polymeric species as a function of their chain length (and hence conversion). Thus, for example in RAFT polymerizations, the addition of polymeric radicals to polymeric RAFT agents is likely to be much less kinetically and thermodynamically favored than the reactions of corresponding low molecular weight species. These previously unforeseen extended chain length effects may help to account for apparent discrepancies between the kinetic and thermodynamic parameters measured for polymeric species, and those measured or calculated for the corresponding low molecular weight compounds. ${ }^{[13]}$

Keywords: polymers $\bullet$ thermodynamics $\cdot$ supramolecular chemistry $\bullet$ cycloadditions $\bullet$ entropic effects

[1] a) X. Chen, M. A. Dam, K. Ono, A. Mal, H. Shen, S. R. Nutt, K. Sheran, F. Wudl, Science 2002, 295, 1698-1702; b) N. K. Guimard, K. K. Oehlenschlaeger, J. Zhou, S. Hilf, F. G. Schmidt, C. Barner-Kowollik, Macromol. Chem. Phys. 2012, 213, 131-143; c) L. M. de Espinosa, G. L. Fiore, C. Weder, E. Johan Foster, Y. C. Simon, Prog. Polym. Sci. 2015, 49-50, 60-78; d) N. Kuhl, S. Bode, R. K. Bose, J. Vitz, A. Seifert, S. Hoeppener, S. J. Garcia, S. Spange, S. van der Zwaag, M. D. Hager, U. S. Schubert, Adv. Funct. Mater. 2015, 25, 3295-3301; e) C. J. Kloxin, C. N. Bowman, Chem. Soc. Rev. 2013, 42, 7161-7173; f) M. Guo, L. M. Pitet, H. M. Wyss, M. Vos, P. Y. Dankers, E. W. Meijer, J. Am. Chem. Soc. 2014, 136, 6969-6977; g) S. Basak, J. Nanda, A. Banerjee, Chem. Commun. 2014, 50, 2356-2359; h) T. Aida, E. W. Meijer, S. I. Stupp, Science 2012, 335, 813-817; i) A. V. Ambade, S. K. Yang, M. Weck, Angew. Chem. Int. Ed. 2009, 48, 2894-2898; Angew. Chem. 2009, 121, 2938-2942; j) O. Altintas, D. Schulze-Suenninghausen, B. Luy, C. Barner-Kowollik, Eur. Polym. J. 2015, 62, 409-417; k) J. Willenbacher, O. Altintas, P. W. Roesky, C. Barner-Kowollik, Macromol. Rapid Commun. 2014, 35, 45-51; I) S. Billiet, K. De Bruycker, F. Driessen, H. Goossens, V. Van Speybroeck, J. M. Winne, F. E. Du Prez, Nat. Chem. 2014, 6, 815-821; m) E. Krieg, H. Weissman, E. Shirman, E. Shimoni, B. Rybtchinski, Nat. Nanotechnol. 2011, 6, 141-146; n) G. I. Peterson, M. B. Larsen, A. J. Boydston, Macromolecules 2012, 45, 7317-7328.

[2] E. H. Feng, W. B. Lee, G. H. Fredrickson, Macromolecules 2007, 40, 693-702.

[3] a) A. E. Tonelli, Comput. Polym. Sci. 1992, 2, 67-71; b) N. K. Guimard, J. Ho, J. Brandt, C. Y. Lin, M. Namazian, J. O. Mueller, K. K. Oehlenschlaeger, S. Hilf, A. Lederer, F. G. Schmidt, M. L. Coote, C. Barner-Kowollik, Chem. Sci. 2013, 4, 2752-2759; c) K. Pahnke, O. Altintas, F. G. Schmidt, C. Barner-Kowollik, ACS Macro Lett. 2015, 774777; d) O. Altintas, K. Riazi, R. Lee, C. Y. Lin, M. L. Coote, M. Wilhelm, C. Barner-Kowollik, Macromolecules 2013, 46, 8079-8091; e) K. Pahnke, 
J. Brandt, G. Gryn'ova, P. Lindner, R. Schweins, F. G. Schmidt, A. Lederer, M. L. Coote, C. Barner-Kowollik, Chem. Sci. 2015, 6, 1061-1074.

[4] a) J. P. A. Heuts, G. T. Russell, Eur. Polym. J. 2006, 42, 3-20; b) B. B. Noble, M. L. Coote, Int. Rev. Phys. Chem. 2013, 32, 467-513.

[5] S. K. Yang, A. V. Ambade, M. Weck, J. Am. Chem. Soc. 2010, 132, $1637-$ 1645.

[6] T. F. A. De Greef, M. J. Kade, K. E. Feldman, E. J. Kramer, C. J. Hawker, E. W. Meijer, J. Polym. Sci., Part A: Polym. Chem. 2011, 49, 4253-4260.

[7] R. S. Macomber, J. Chem. Educ. 1992, 69, 375-378.

[8] a) J. Brandt, N. Guimard, C. Barner-Kowollik, F. Schmidt, A. Lederer, Anal. Bioanal. Chem. 2013, 405, 8981-8993; b) J. Brandt, K. K. Oehlenschlaeger, F. G. Schmidt, C. Barner-Kowollik, A. Lederer, Adv. Mater. 2014, 26, 5758-5785.

[9] D. Berek, J. Chromatogr. A 2002, 950, 75-80.
[10] a) Roestamsjah, L. A. Wall, R. E. Florin, M. H. Aldridge, L. J. Fetters, J Res. Nat. Bur. Stand. 1978, 83, 371-380; b) J. Ulbricht, R. Jordan, R. Luxenhofer, Biomaterials 2014, 35, 4848-4861.

[11] R. Lee, M. L. Coote, PCCP 2013, 15, 16428-16431.

[12] a) B. M. E. van der Hoff, C. E. Gall, J. Macromol. Sci. Part A Pure Appl. Chem. 1977, 11, 1739-1758; b) G. J. Price, P. F. Smith, Polym. Int. 1991, 24, $159-164$.

[13] a) C. Barner-Kowollik, Handbook of RAFT Polymerization, Wiley-VCH, Weinheim, Germany, 2008; b) C. Barner-Kowollik, M. Buback, B. Charleux, M. L. Coote, M. Drache, T. Fukuda, A. Goto, B. Klumperman, A. B. Lowe, J. B. McLeary, G. Moad, M. J. Monteiro, R. D. Sanderson, M. P. Tonge, P. Vana, J. Polym. Sci., Part A: Polym. Chem. 2006, 44, 5809-5831; c) T. Junkers, C. Barner-Kowollik, M. L. Coote, Macromol. Rapid Commun. 2011, 32, 1891-1898. 
Middle vs. End: We show that, all other conditions being equal, bond cleavage in the middle of molecules is much more favored compared to bond cleavage at the end. Experimental and theoretical approaches have been used to study the selectivity of bond cleavage or dissociation of both covalent and supramolecular adducts. The extensive implications for other fields of chemistry are discussed.
Kai Pahnke, Josef Brandt, Ganna Gryn'ova, Ching Y. Lin, Ozcan Altintas, Friedrich G. Schmidt, Albena Lederer, ${ }^{*}$ Michelle L. Coote, * and Christopher Barner-Kowollik*

Entropy-Driven Selectivity for Chain Scission: Where Macromolecules Cleave 